\title{
LA-UR-12-25417
}

Approved for public release; distribution is unlimited.

Title:

Author(s):

Intended for:
NDI Multigroup Radiochemistry Dosimetry Library hoffman2009

Lee, Mary B

Interlab communication

Disclaimer:

Los Alamos National Laboratory, an affirmative action/equal opportunity employer,is operated by the Los Alamos National

Security, LLC for the National NuclearSecurity Administration of the U.S. Department of Energy under contract DE-AC52-06NA25396. By approving this article, the publisher recognizes that the U.S. Government retains nonexclusive, royalty-free license to publish or reproduce the published form of this contribution, or to allow others to do so, for U.S. Government purposes.

Los Alamos National Laboratory requests that the publisher identify this article as work performed under the auspices of the

U.S. Departmentof Energy. Los Alamos National Laboratory strongly supports academic freedom and a researcher's right to publish; as an institution, however, the Laboratory does not endorse the viewpoint of a publication or guarantee its technical correctness. 
Los Alamos NATIONAL LABORATORY EST. 1943

memorandum

Computational Physics

$X C P-5$
To/MS: Joann M. Campbell, T087

From/Ms: Mark G. Gray, F663

Mary Beth Lee, F663

Phone/FAX: $\quad 7-5341,5-2879$

Symbol: $\quad \mathrm{XCP}-5: 12-31(\mathrm{U})$

Date: October 3, 2012

\section{NDI Multigroup Radiochemistery Dosimetry Library hoffman2009}

\section{Summary}

We have processed the modeled neutron induced nuclear reaction cross section data from Hoffman et al.[1] into NDI 618_lanl multigroup neutron dosimetry tables for yttrium, zirconium, and rhodium, and created corresponding radiochemistry chain files. The resulting cross sections for ${ }^{87 \mathrm{~g}} \mathrm{Y}(\mathrm{n}, \gamma)^{88 \mathrm{~g}} \mathrm{Y}$ and ${ }^{88 \mathrm{~g}} \mathrm{Y}(\mathrm{n}, 2 \mathrm{n})^{87 \mathrm{~g}} \mathrm{Y}$ compare favorably to the data in the $\mathrm{rc2004} 618$ library and are similar to the data in the crsln $\mathrm{c}$ library. We have released these data and chains to facilitate the direct comparison of yttrium ratios for Los Alamos and Livermore data and request feedback from our user community on the usefulness of this data before we process more data.

\section{Background}

Since the release of the high fidelity transport data in NDI 2.0.3 (Fall 2005), our users have requested radiochemistry data in the 618_lanl group structure. In response we released the rc2 004 4618[2] library in NDI 2.0.4 (Spring 2006) containing data for the tracers yttrium, thulium, and iridium, followed by updates to the iridium cross sections in the rc2006_618 library (Spring 2007). Unfortunately these three high quality radiochemistry tracers have not satisfied our users who are accustomed to the dozens of low quality, low fidelity radiochemistry tracers in our historic crsdata library.

Recently (Summer 2012) the nuclear data team obtained a $\mathrm{CD}^{1}$ of modeled radiochemistry data from Livermore[1]. This memo documents our evaluation of that data, our processing it into NDI multigroup neutron dosimetry data, our comparison to NDI existing production neutron dosimetry data, and our creation of radiochemistry chain data as part of the hof fman 2009 library production release.

\footnotetext{
${ }^{1}$ Special thanks to the nuclear data fairy!
} 


\section{Discussion}

In June 2006 Hoffman et al.[1] documented a set of nuclear reaction cross sections for strontium through rhodium, created by a Hauser-Feshbach model[3] using input parameters created from the local systematic analysis of experimental data. These data are part of their efforts to update and improve Livermore radiochemistry cross section detector sets. The Hoffman 2009 data covers eight neutron induced reactions, four proton induced reactions, and four deuteron induced reactions, on two or three isomer states per isotope, with over a dozen isotopes per tracer element, on a 351 point non-uniform energy grid from $10^{-5}-20 \mathrm{MeV}$, with a data floor at $10^{-8}$ barns. The exact details of the model code they used, the input parameters they developed, the reactions and isomers modeled, and the comparison of their results to experimental data can be found in their paper.

In order to evaluate their data we decided to focus on neutron induced yttrium reactions because we could directly compare it to our data in the rc2004_618 library. We extracted all of the cross sections for yttrium from the Hoffman data set, and coplotted 20 yttrium targets at each of the eight reactions. The plots revealed bad data at the beginning of the ${ }^{89 m} \mathrm{Y}(\mathrm{n}, 3 \mathrm{n})^{87 \mathrm{~m}} \mathrm{Y}$ cross section, which we fixed. By plotting the data with lines on lin-lin, lin-log, log-lin, and log-log scales, we determined that the data was best characterized with a log-log interpolation between the points, which would prove useful when we later had to analytically extend the data.

Figure 1 shows four typical neutron induced continuous energy non-threshold reaction cross sections from their work: ${ }^{88 \mathrm{~m}} \mathrm{Y}\left(\mathrm{n}, \mathrm{n}\right.$ ' ${ }^{88 \mathrm{~g}} \mathrm{Y},{ }^{87 \mathrm{~g}} \mathrm{Y}(\mathrm{n}, \gamma)^{88 \mathrm{~g}} \mathrm{Y},{ }^{88 \mathrm{~g}} \mathrm{Y}(\mathrm{n}, \mathrm{p})^{88 \mathrm{~g}} \mathrm{Sr}$, and ${ }^{87 \mathrm{~g}} \mathrm{Y}(\mathrm{n}, \alpha)^{84 \mathrm{~g}} \mathrm{Rb}$. This data is typical of all the non-threshold data we processed from the Hoffman 2009 data set, with their data floor occasionally cutting off the cross sections, as seen here in the (n, $\alpha)$ reaction, and with a low energy asymptotic $1 / v$ form.

Figure 2 shows four typical neutron induced continuous energy threshold reaction cross sections from their work: ${ }^{88 \mathrm{~g}} \mathrm{Y}(\mathrm{n}, 2 \mathrm{n})^{87 \mathrm{~g}} \mathrm{Y},{ }^{90 \mathrm{~m}} \mathrm{Y}(\mathrm{n}, 3 \mathrm{n})^{88 \mathrm{~g}} \mathrm{Y},{ }^{87 \mathrm{~g}} \mathrm{Y}(\mathrm{n}, \mathrm{np})^{86 \mathrm{~g}} \mathrm{Sr}$, and ${ }^{87 \mathrm{~g}} \mathrm{Y}(\mathrm{n}, \mathrm{n} \alpha)^{83 \mathrm{~g}} \mathrm{Rb}$. This data is typical of all the threshold data we processed from the Hoffman 2009 data set, with their floor defining the "zero" below threshold for these reactions.

All the Hoffman 2009 reactions we examined had reasonable shapes and magnitudes, but the Hauser-Feshbach model with experimental fit did not, and indeed cannot, capture the fine structures expected in the actual cross sections.

To process this data into multigroup format we had to determine an appropriate interpolation scheme to use on their 351 point energy grid, analytically extend the data to the 618_lan l energy range, and compute the TD weighted average of the cross sections. Our plots of the continuous energy data suggested log-log interpolation, and this made the analytic extension of the Hoffman 2009 continuous energy data from $10^{-5} \mathrm{MeV}$ down to $10^{-11} \mathrm{MeV}$ easy: we extended non-threshold cross sections by adding the point $\left(10^{-11} \mathrm{MeV}, 10^{3} y_{1}\right)^{2}$ before $\left(10^{-5} \mathrm{MeV}, y_{1}\right)$; we extended threshold reactions by adding the point $\left(10^{-11} \mathrm{MeV}, 10^{-8}\right.$ barns $)$ before $\left(10^{-5} \mathrm{MeV}, 10^{-8}\right.$ barns $)$. The analytically extended continuous energy cross sections were then averaged in the 618_lanl en-

\footnotetext{
${ }^{2} \sigma(E) \sim 1 / v$ implies a log-log interpolation line with slope $\left.-\frac{1}{2}=\frac{\log \left(y_{0}\right)-\log \left(y_{1}\right)}{\log \left(10^{-11}\right)-\log \left(10^{-5}\right.}\right)$ which implies $y_{0}=10^{3} y_{1}$.
} 


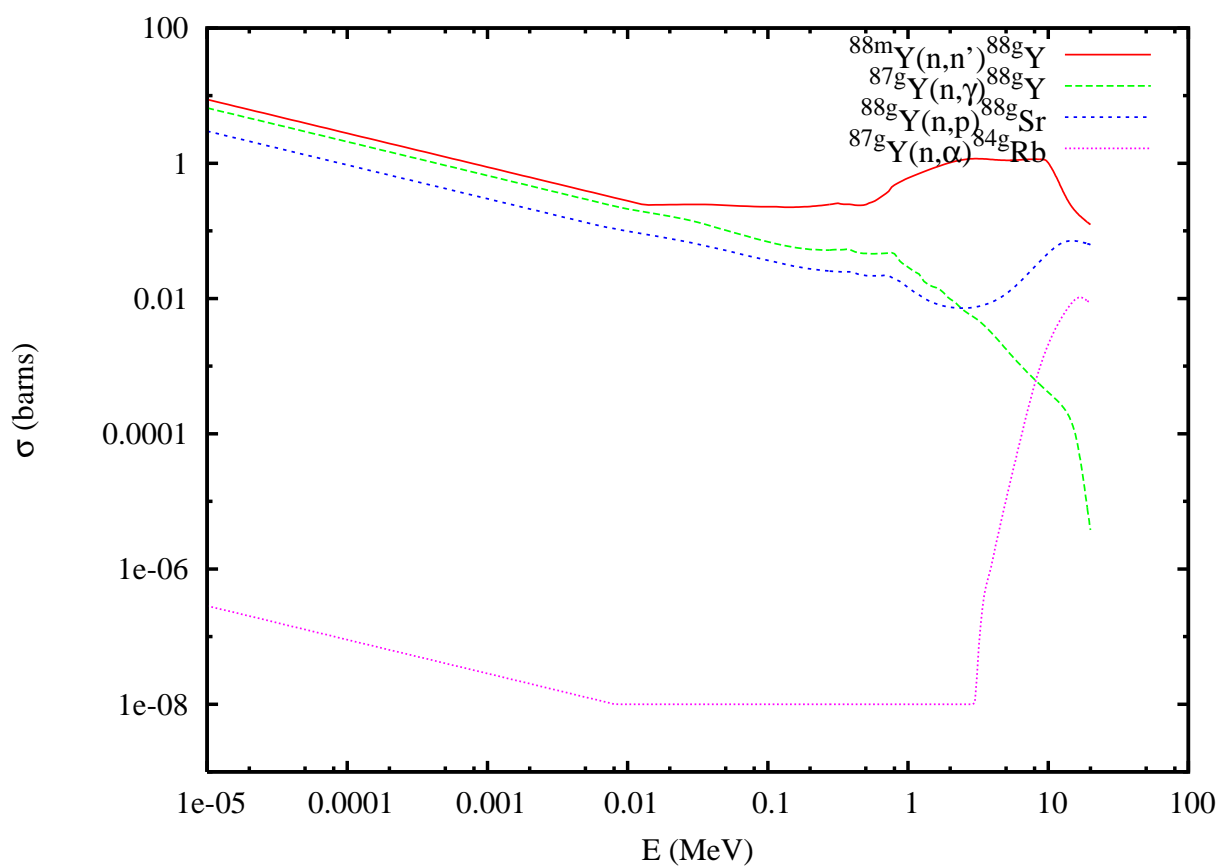

Figure 1: The ${ }^{88 \mathrm{~m}} \mathrm{Y}\left(\mathrm{n}, \mathrm{n}^{\prime}\right)^{88 \mathrm{~g}} \mathrm{Y},{ }^{87 \mathrm{~g}} \mathrm{Y}(\mathrm{n}, \gamma)^{88 \mathrm{~g}} \mathrm{Y},{ }^{88 \mathrm{~g}} \mathrm{Y}(\mathrm{n}, \mathrm{p})^{88 \mathrm{~g}} \mathrm{Sr}$, and ${ }^{87 \mathrm{~g}} \mathrm{Y}(\mathrm{n}, \alpha)^{84 \mathrm{~g}} \mathrm{Rb}$ continuous energy cross sections from the Hoffman 2009 data set. Their data floor at $10^{-8}$ barns truncates the the $(\mathrm{n}, \alpha)$ cross section reaction between 0.01 and $3 \mathrm{MeV}$.

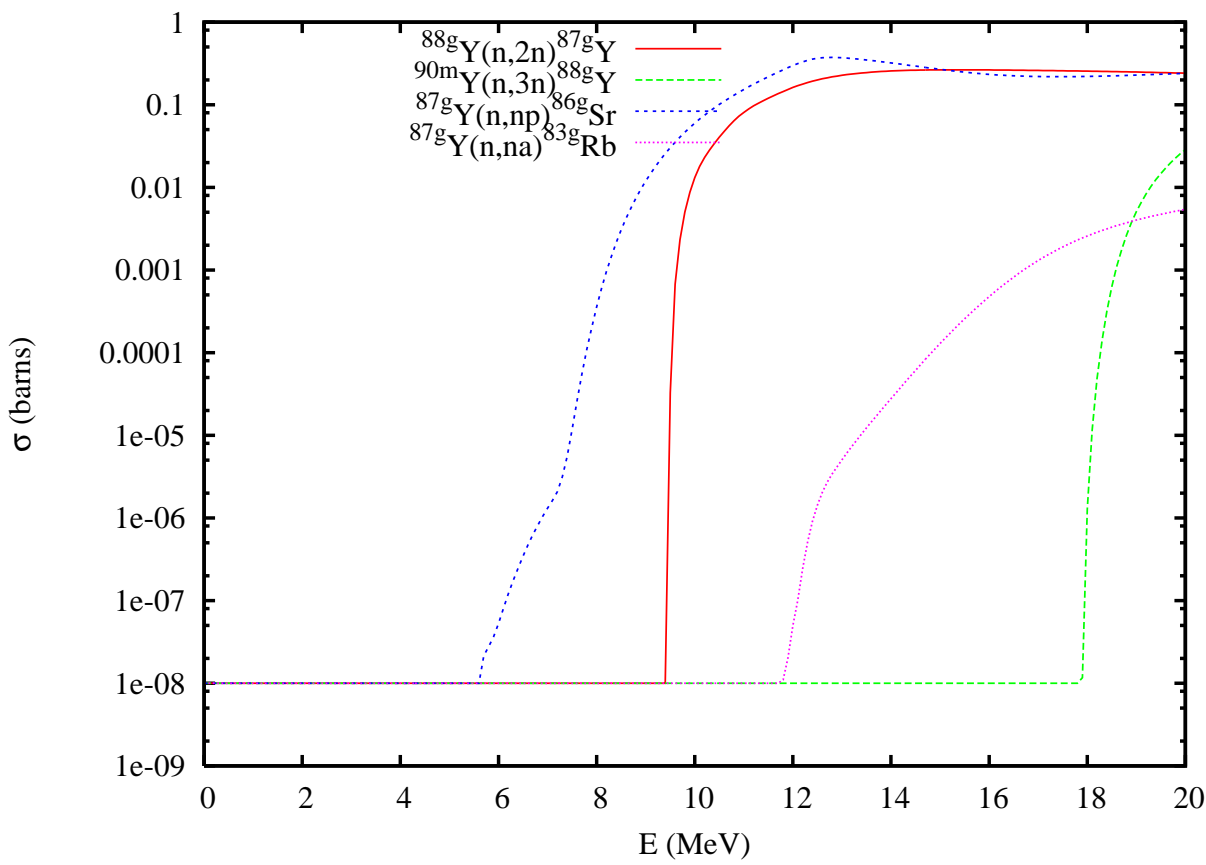

Figure 2: The ${ }^{88 \mathrm{~g}} \mathrm{Y}(\mathrm{n}, 2 \mathrm{n})^{87 \mathrm{~g}} \mathrm{Y},{ }^{90 \mathrm{~m}} \mathrm{Y}(\mathrm{n}, 3 \mathrm{n})^{88 \mathrm{~g}} \mathrm{Y},{ }^{87 \mathrm{~g}} \mathrm{Y}(\mathrm{n}, \mathrm{np})^{86 \mathrm{~g}} \mathrm{Sr}$, and ${ }^{87 \mathrm{~g}} \mathrm{Y}(\mathrm{n}, \mathrm{n} \alpha)^{83 \mathrm{~g}} \mathrm{Rb}$ continuous energy cross sections from the Hoffman 2009 data set. 
ergy boundaries with the room temperature TD weight function[5]. Since the 618_lanl group intervals are small, quadrature was approximated with a 10 point Gauss-Legendre scheme.

Figure 3 shows the ${ }^{87 g} Y(n, \gamma)^{88 g} Y$ multigroup energy cross sections from the hof fman 2009 and rc2004_618 in the 618_lanl group structure.

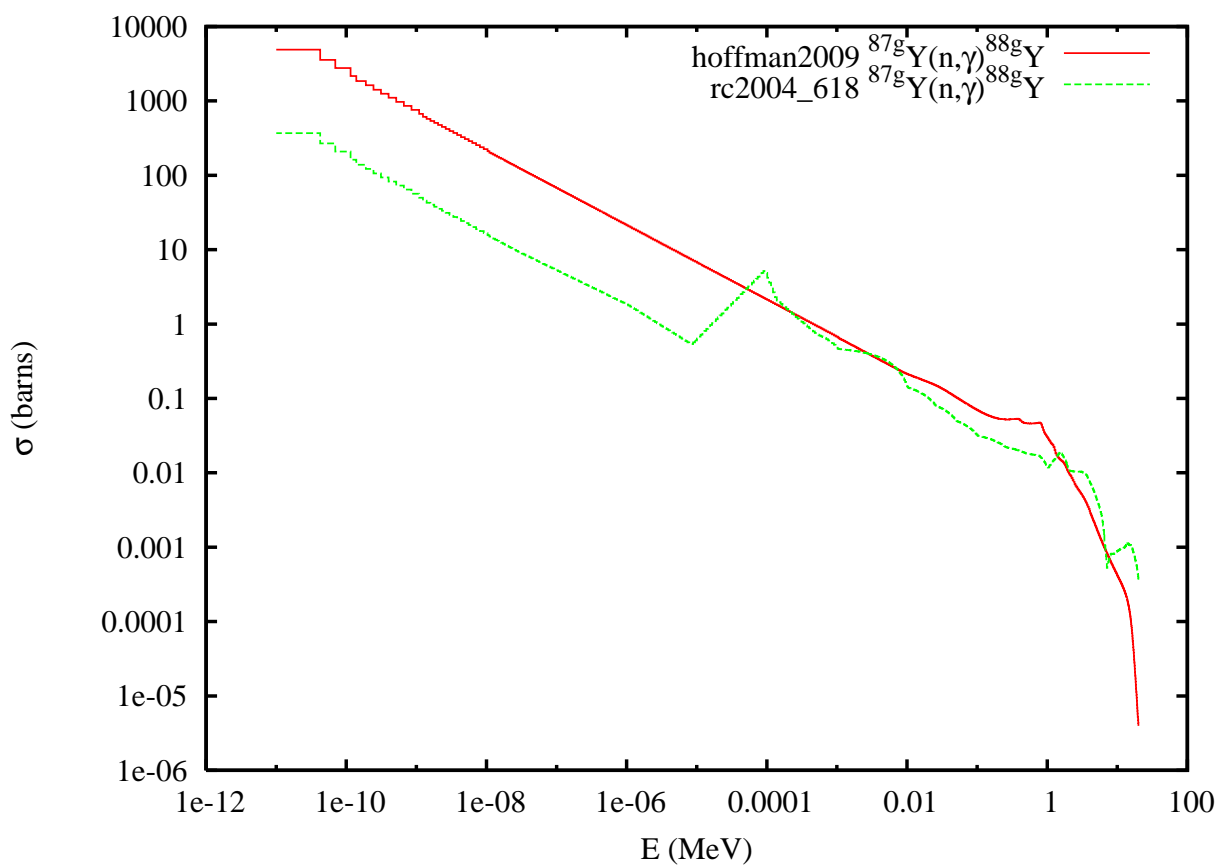

Figure 3: The ${ }^{87 \mathrm{~g}} \mathrm{Y}(\mathrm{n}, \gamma)^{88 \mathrm{~g}} \mathrm{Y}$ multigroup energy cross sections from the hoffman2009 and rc2004_618 NDI libraries in 618 groups. The Hoffman continuous energy data was analytically extended with a $1 / v$ fit and averaged with the TD weight function.

As expected, the rc2004_618 data shows more structure than the hoffman2009 data, particularly in the mid and high energy regions. Still, given the typical uncertainties in (n, $\gamma)$ cross sections, the hoffman2009 data is reasonable.

Figure 4 shows the ${ }^{88 \mathrm{~g}} \mathrm{Y}(\mathrm{n}, 2 \mathrm{n})^{87 \mathrm{~g}} \mathrm{Y}$ multigroup energy cross sections from the hof fman 2009 and rc2004_618 in the 618_lanl group structure.

Here the hoffman2009 data is a good match to the rc2004_618 data, except, of course for the expected mismatch below the artificial floor.

As a further check on the multigroup data, we compared the sum of cross section to specific isomers to the total cross section for each reaction. The threshold reactions passed this test except, of course, for the floored values. The non-threshold reactions passed this test at high energies, but any low energy tail which came from a minimum below the floor did not. Unfortunately this "feature", which comes directly from the raw data, means that some of the non-threshold reactions to specific isomeric states have a low energy tail that is too big. Fortunately this affects primarily the less important $(\mathrm{n}, \mathrm{p})$ and $(\mathrm{n}, \alpha)$ reactions. 


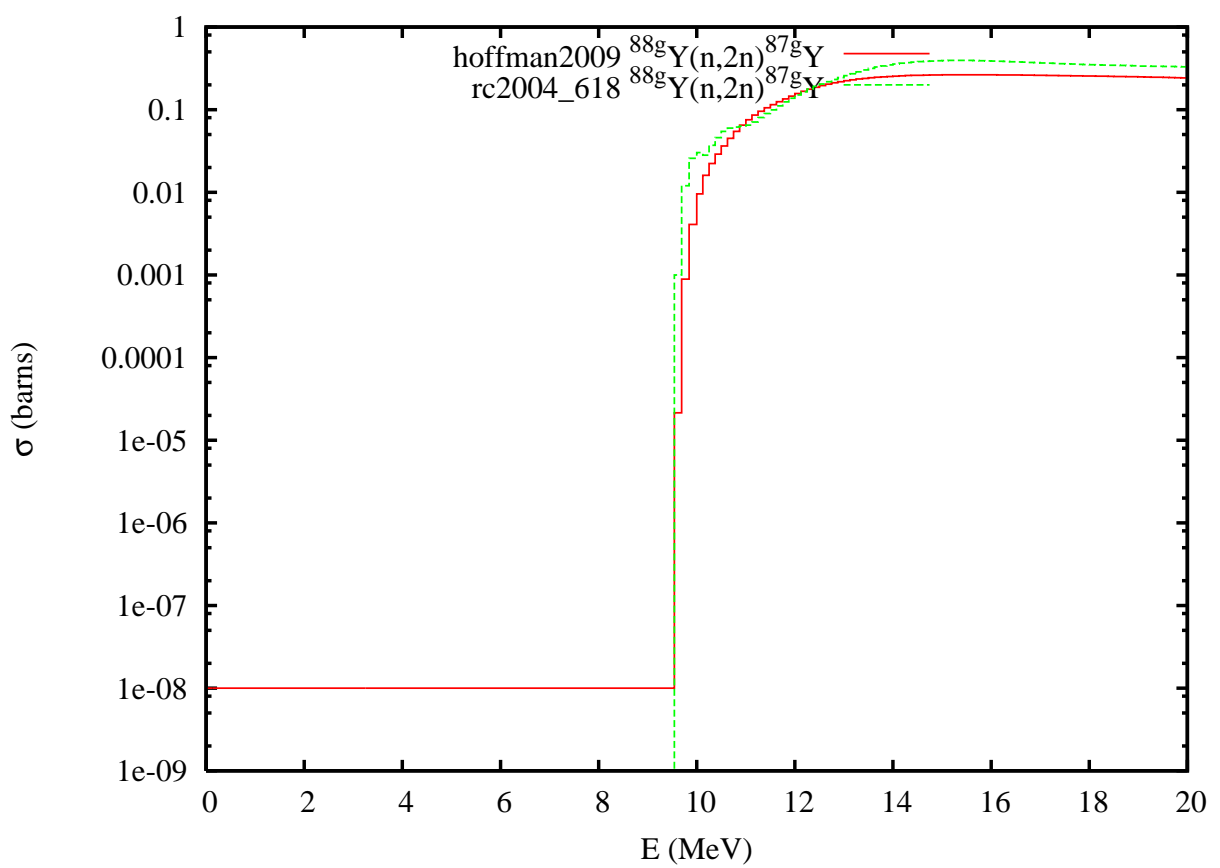

Figure 4: The ${ }^{88 \mathrm{~g}} \mathrm{Y}(\mathrm{n}, 2 \mathrm{n})^{87 \mathrm{~g}} \mathrm{Y}$ multigroup energy cross sections from hoffman2009 and rc2004_618 NDI libraries in 618 groups. The Hoffman continuous energy data was analytically extended with a constant fit and averaged with the TD weight function.

A better characterization of the hoffman2009 data is its comparison of it to the most popular NDI neutron dosimetry libraries. Figure 5 shows the ${ }^{87 \mathrm{~g}} \mathrm{Y}(\mathrm{n}, \gamma)^{88 \mathrm{~g}} \mathrm{Y}$ multigroup energy cross sections from the hoffman2009, t16_rc_2004, crsllnl, and y21grp in the 21_lanl group structure.

The t16_rc_2004 data represents our best neutron dosimetry data in the 30_lanl group structure (it is the basis for the rc2004_618 library), while y21grp is an older LANL neutron dosimetry library consistent with our crsdata set. The crsllnl library data represents an older LLNL neutron dosimetry set in NDI format. The agreement is again good given the traditional uncertainties in $(n, \gamma)$ reaction data. The two LANL data sets show a high energy peak, while the two LLNL data sets lack this feature.

Figure 6 shows the ${ }^{88 \mathrm{~g}} \mathrm{Y}(\mathrm{n}, 2 \mathrm{n})^{87 \mathrm{~g}} \mathrm{Y}$ multigroup energy cross sections from the hof fman 2009 , t16_rc_2004, crsllnl, and y2lgrp in the 21_lanl group structure.

The four sets show good agreement at high energies, but both LLNL sets have a lower cross section in the $10-12 \mathrm{MeV}$ range.

In order to produce NDI neutron dosimetry tables, we had to supplement the Hoffman 2009 cross section data data with additional nuclear properties culled from ENDF-VII.0[4] file MF=1, section MT=451. The processing work flow is shown in Appendix I. In addition to the yttrium neutron dosimetry tables we processed for comparison purposes we also created a reaction chain, shown 


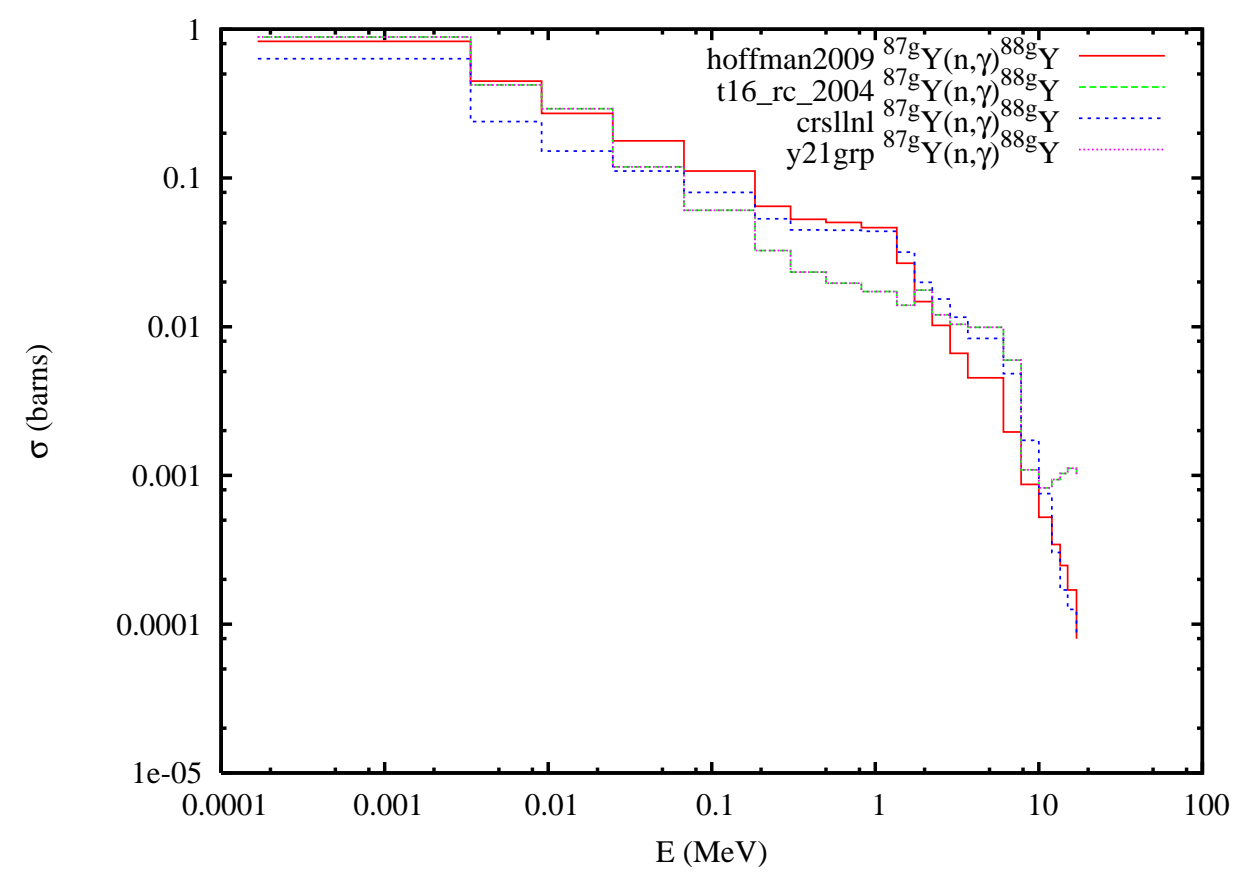

Figure 5: The ${ }^{87 g} \mathrm{Y}(\mathrm{n}, \gamma)^{88 \mathrm{~g}} \mathrm{Y}$ multigroup energy cross sections from hoffman2009, t16_rc_2004, crsllnl, and y21grp NDI libraries in 21 groups.

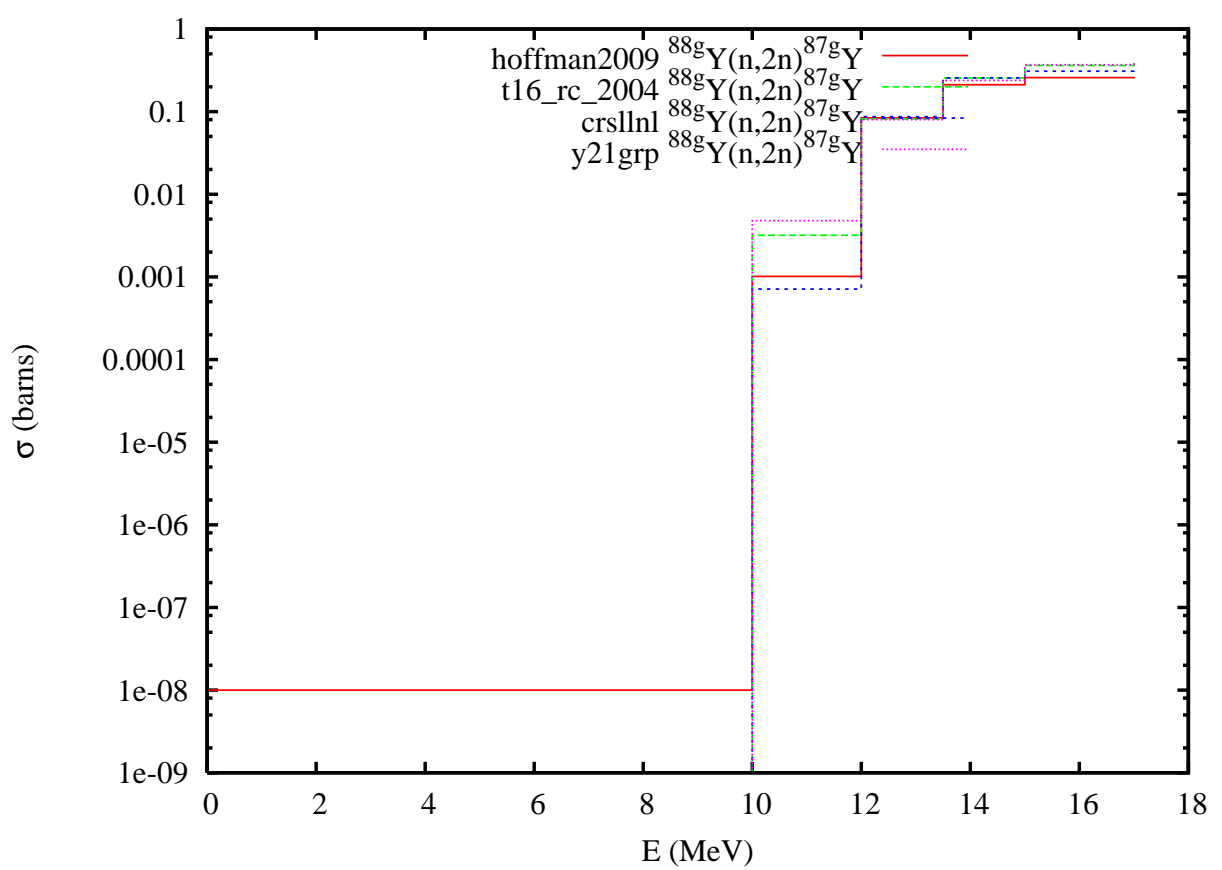

Figure 6: The ${ }^{88 \mathrm{~g}} \mathrm{Y}(\mathrm{n}, 2 \mathrm{n})^{87 \mathrm{~g}} \mathrm{Y}$ multigroup energy cross sections from hoffman2009, t16_rc_2004, crsllnl, and y21grp NDI libraries in 21 groups. 
in Appendix II. We also processed zirconium, with representative plot in Figure 7 and chain in Appendix III, and rhodium, with representative plot in Figure 8 and chain in Appendix IV, which have been requested by our user community. 


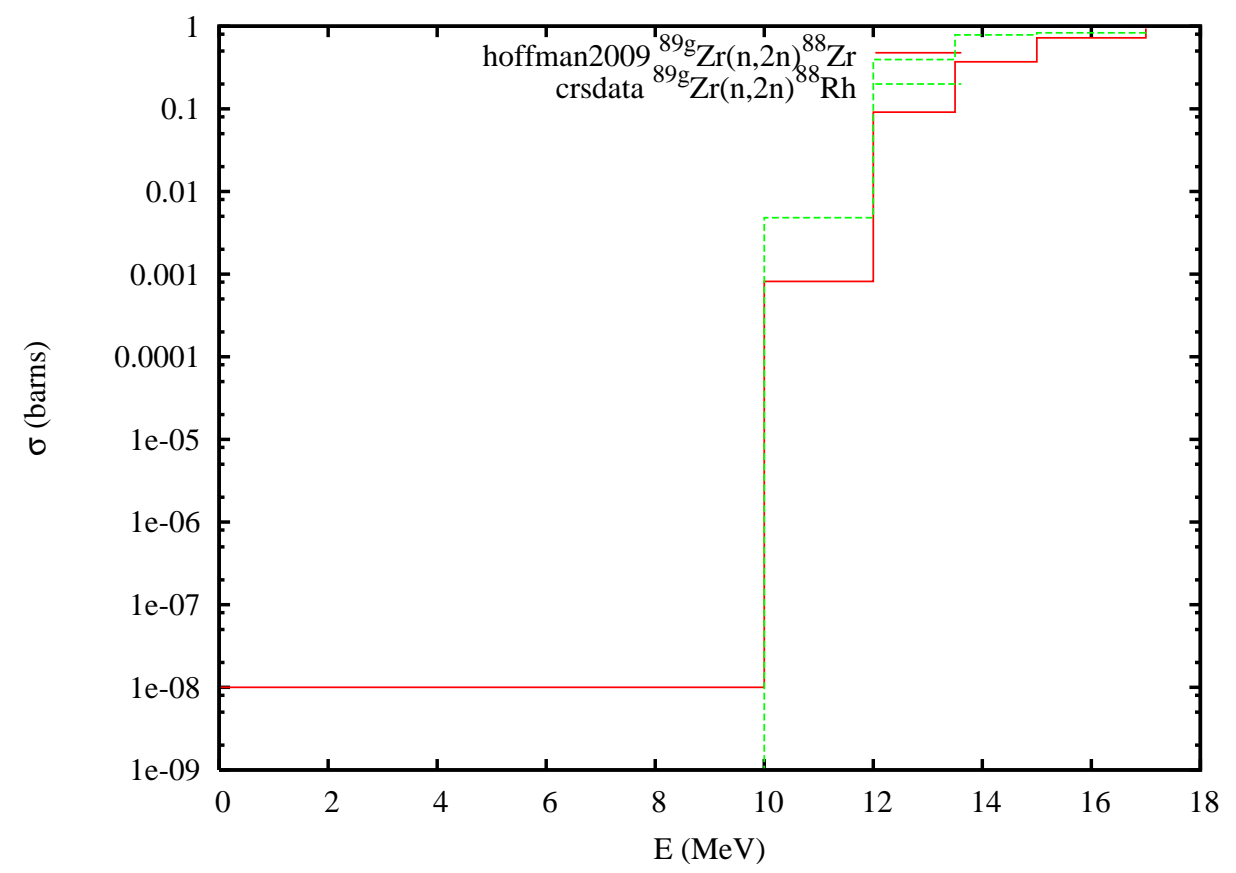

Figure 7: The ${ }^{89 g} \mathrm{Zr}(\mathrm{n}, 2 \mathrm{n})^{88} \mathrm{Zr}$ multigroup energy cross sections from hoffman2009 and crsdata NDI libraries in 30 groups.

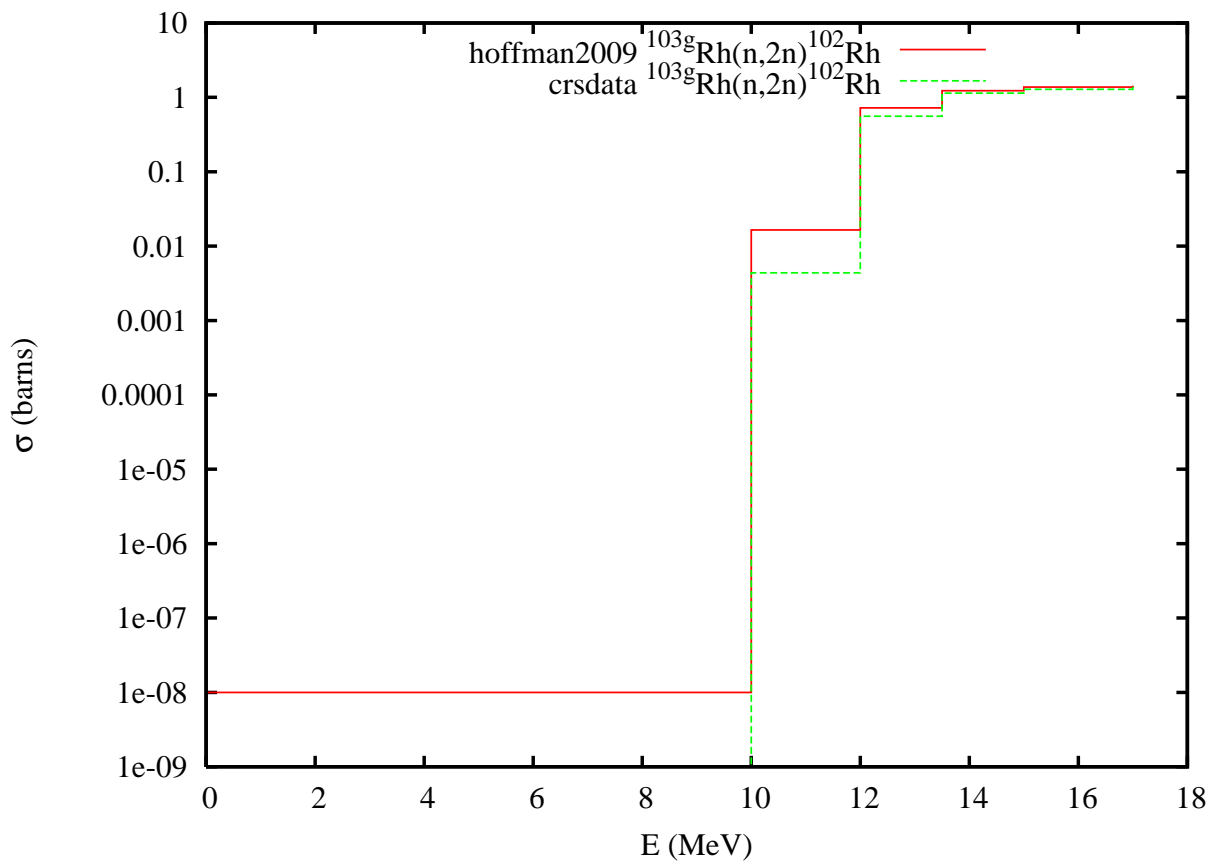

Figure 8: The ${ }^{103 g} \mathrm{Rh}(\mathrm{n}, 2 \mathrm{n})^{102} \mathrm{Rh}$ multigroup energy cross sections from hof fman 2009 and crsdata NDI libraries in 30 groups. 


\section{Conclusion}

We have processed the modeled neutron induced nuclear reaction cross section data from Hoffman et al. in NDI 618_lan l multigroup neutron dosimetry tables for yttrium, zirconium, and rhodium. The resulting yttrium cross sections compare favorably with the data in the rc2004_618 library and resemble the data in the crsllnl library. We request feedback from our user community on the usefulness of this data by the direct comparison of it to our yttrium data; based on their response we will proceed to process more of the Hoffman 2009 data.

Although the Hoffman data is not as structured as our best neutron dosimetry data, it does cover more isotopes per element and more isomers per isotope. It might be interesting to study the effects of the extended chains versus the effects of more structured data. Further, by covering a range of isotopes, the Hoffman data offers the possibility of modeling cross tracer isotopics: for example the neutron induced zirconium cross sections contain (n,p) and (n,np) reactions that could link it to the yttrium chain, and the proton induced reaction cross sections on yttrium, which we did not process, could link it through the $(\mathrm{p}, \mathrm{n})$ and $(\mathrm{p}, 2 \mathrm{n})$ reactions to the zirconium chain. As we get more information on the quality of this data, we can proceed with the processing of additional data.

The Hoffman 2009 data is one of a series of LLNL systematic evaluations of dosimetry data; this work has established a path for our further processing of that data if this data proves useful.

\section{References}

[1] R. D. Hoffman, K. Kelley, F. S. Dietrich, R. Bauer, and M. G. Mustafa, Modeled Neutron and Charged-Particle Induced Nuclear Reaction Cross Sections for Radiochemistry in the Region of Yttrium, Zirconium, Niobium, and Molybdenum, UCRL-TR-222275, June 20, 2006

[2] H. Trellue and M. White, Release of New NDI Library RC2004_618: Radiochemistry Data in 618 Groups, X-1:HT-06-08, February 15, 2006

[3] W. Hauser and H. Feshbach, The Inelastic Scattering of Neutrons, Phys. Rev. 87, 366, 1952

[4] M. B. Chadwick et al., ENDF/VII.0: Next Generation Evaluated Nuclear Data Library for Nuclear Science and Technology, Nuclear Data Sheets, 107, 2931-3060, 2006

[5] Morgan C. White, The TD Weight Function Revisited, X-5:05-24(U), March 21, 2005 


\section{Appendix I: hoffman2009 Processing Work Flow}

The single border boxes represent primary data sources, the double border boxes represent derived data, and the triple border box represents the final product (hoffman2009 neutron dosimetry files). The ovals represent processing, plotting, and checking codes.

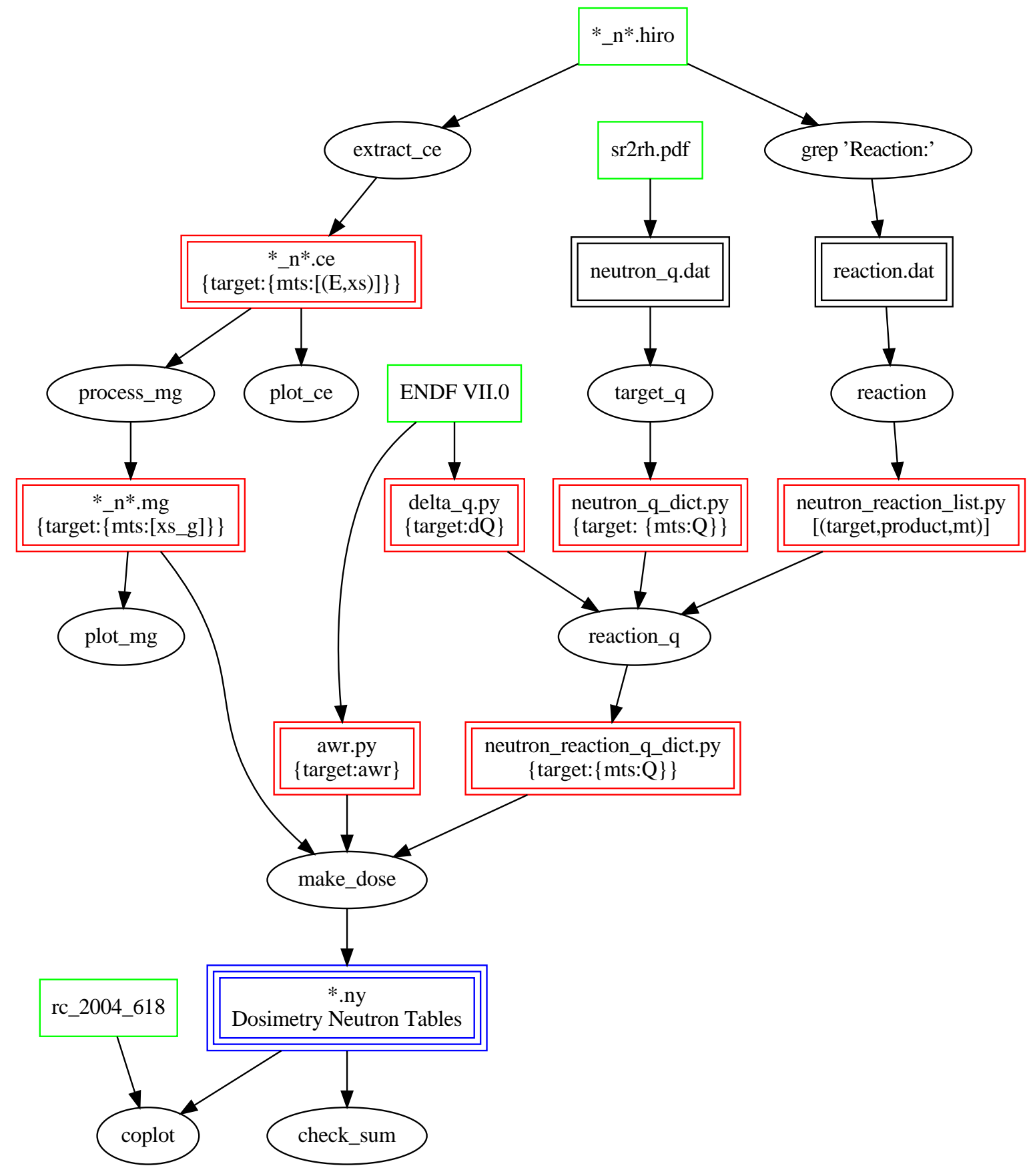




\section{Appendix II: hoffman2009 Yttrium Chain}

The red arrows represent $(n, 3 n)$ reactions. The green lines represent $(n, \gamma)$ and $(n, 2 n)$ pairs. The black lines represent (n,n') reactions. The circled isomers are the targets in the rc2004_618 neutron dosimetry library.

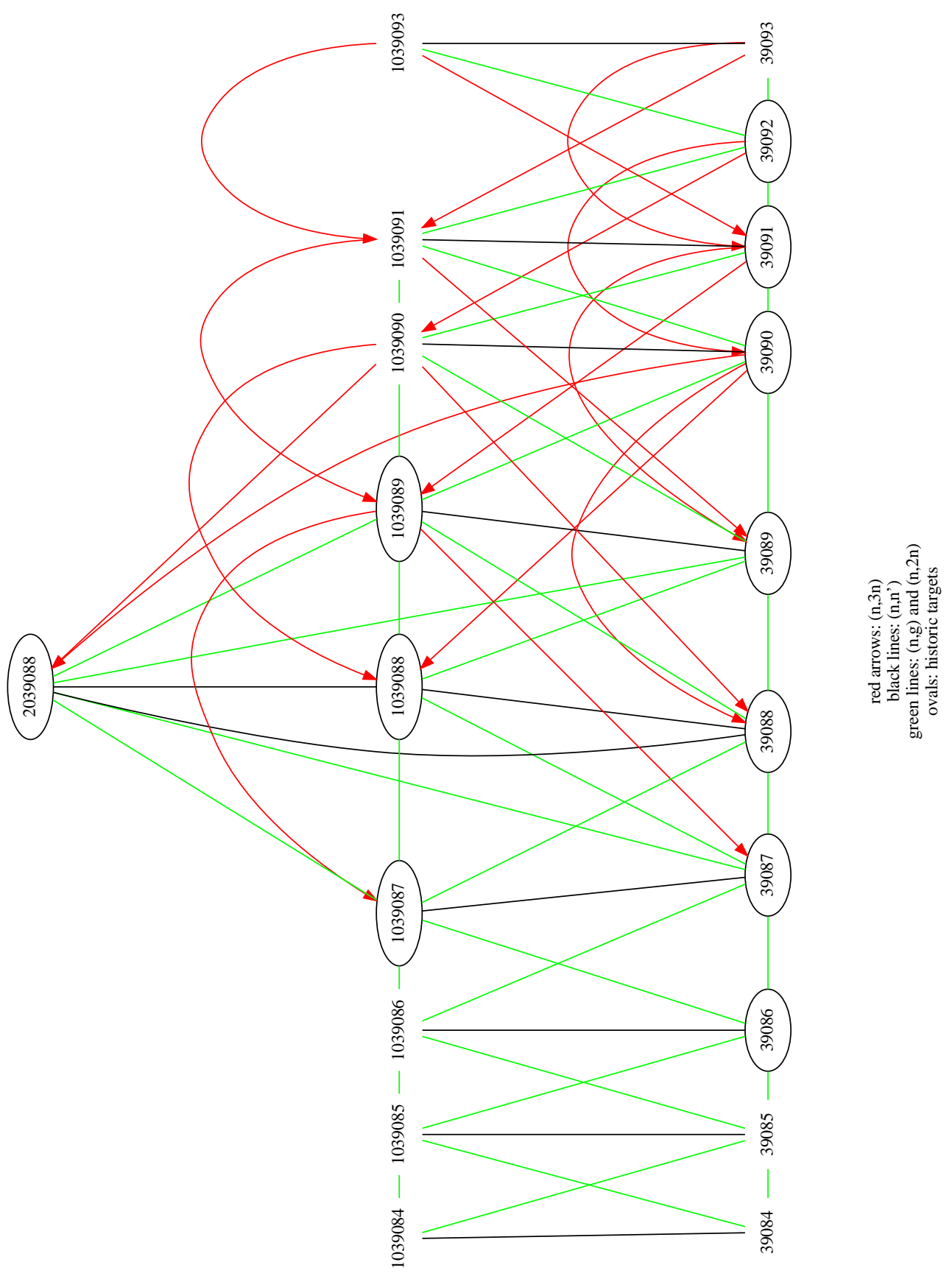




\section{Appendix III: hoffman2009 Zirconium Chain}

The red arrows represent $(n, 3 n)$ reactions. The green lines represent $(n, \gamma)$ and $(n, 2 n)$ pairs. The black lines represent (n,n') reactions. The circled isomers are the targets in the crsdata neutron dosimetry library.

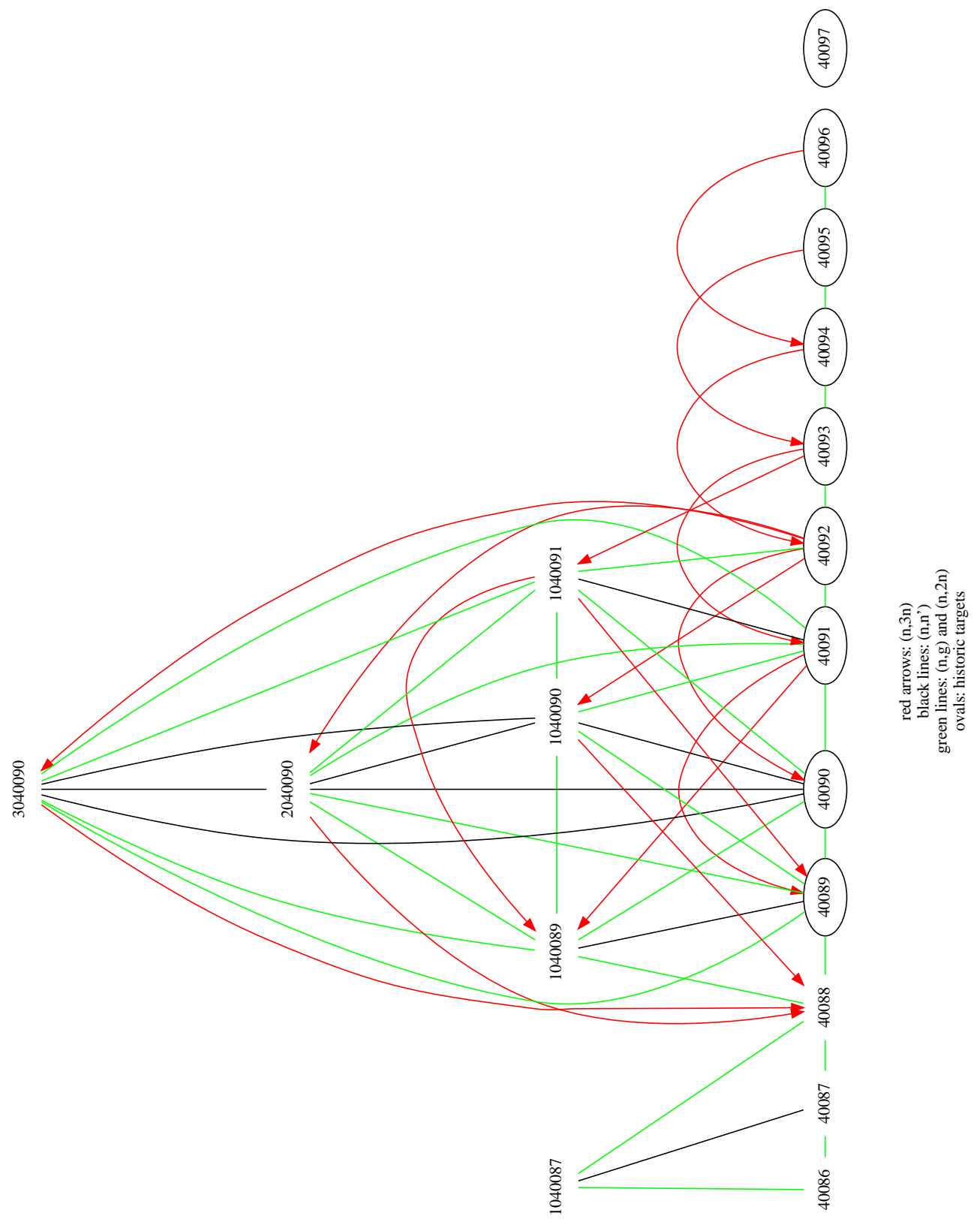




\section{Appendix IV: hoffman2009 Rhodium Chain}

The red arrows represent (n,3n) reactions. The green lines represent $(n, \gamma)$ and $(n, 2 n)$ pairs. The black lines represent (n,n') reactions. The circled isomer is the only target in the crs data neutron dosimetry library.
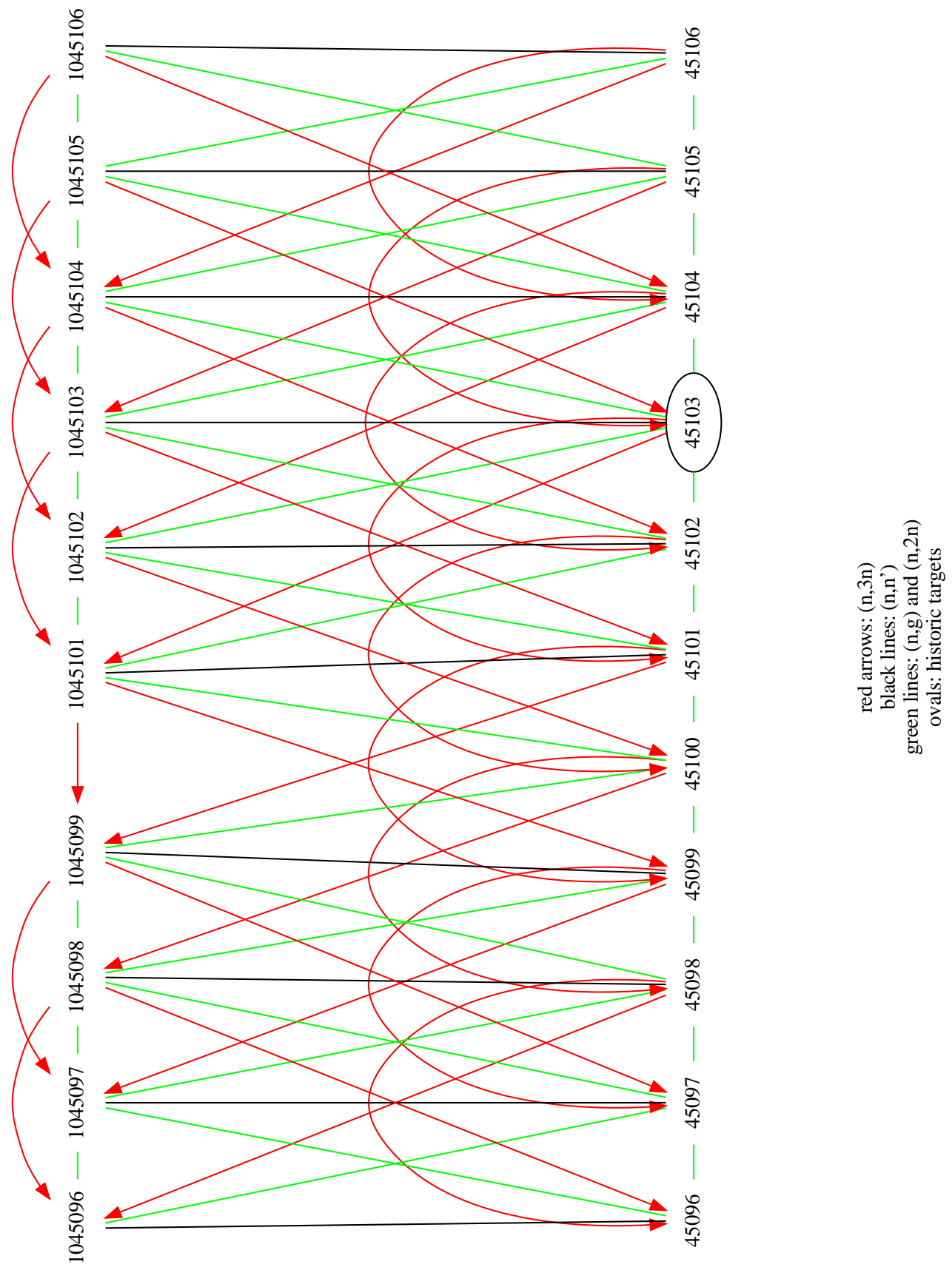
$\mathrm{XCP}-5: 12-31(\mathrm{U})$

MGG:mgg

ADC:dkp

Distribution:

NDI users

XCP-5 File 